\title{
First trimester angiogenic and inflammatory factors in women with chronic hypertension and impact of blood pressure control: a case-control study.
}

\author{
Diane Nzelu ${ }^{1}$, Kypros Nicolaides ${ }^{2}$, and Nikos Kametas ${ }^{3}$ \\ ${ }^{1}$ KINGS COLLEGE HOSPITAL NHS TRUST \\ ${ }^{2}$ Fetal Medicine Institute \\ ${ }^{3}$ Kings College Hospital
}

November 11, 2020

\begin{abstract}
Objectives: Assess first trimester serum placental growth factor (PLGF), soluble fms-like tyrosine kinase-1 (sFLT-1), interleukin6 (IL-6), tumour necrosis factor- $\alpha(\mathrm{TNF}-\alpha)$, endothelin and vascular cell adhesion molecule (VCAM) in women with chronic hypertension $(\mathrm{CH})$ stratified according to blood pressure (BP) control. Design: Case-control. Setting: Tertiary referral centre. Population: 650 women with CH, 142 normotensive controls. Methods: In the first trimester, patients with CH were subdivided into 4 groups. Group 1 included women without pre-pregnancy CH presenting with BP [?]140/90mmHg. Groups 2-4 had pre-pregnancy $\mathrm{CH}$; in group 2 the $\mathrm{BP}$ was $<140 / 90 \mathrm{mmHg}$ without antihypertensive medication, in group 3 the $\mathrm{BP}$ was $<140 / 90 \mathrm{mmHg}$ with antihypertensive medication and in group 4 the BP was [?] 140/90 $\mathrm{mmHg}$ despite antihypertensive medication. PLGF, sFLT-1, IL-6, TNF- $\alpha$, endothelin and VCAM were measured at $11+0-13+6$ weeks' and converted into multiples of the expected median (MoM) using multivariate regression analysis in the controls. Main outcome measure: Comparisons of MoM values of PLGF, sFLT-1, IL-6, TNF- $\alpha$, endothelin and VCAM between the 4 CH groups and the controls were made using analysis of variance or Kruskal-Wallis tests. Results: In the CH groups, compared to controls, PLGF was reduced in groups 2-4, sFLT-1 was reduced in groups 2 and 3, endothelin was increased in groups 1 and 4 but IL-6 was reduced in group 4. Conclusion: In women with $\mathrm{CH}$, differences exist in first trimester angiogenic and inflammatory profiles according to $\mathrm{BP}$ control. Further evaluation is needed to determine if these differences are useful in the stratification of care.
\end{abstract}

\section{INTRODUCTION}

Chronic hypertension $(\mathrm{CH})$ complicates 1-2\% of pregnancies and represents one of the most significant risk factors for the development of preeclampsia (PE). ${ }^{1}$ We have proposed that women with $\mathrm{CH}$ fall into four categories in relation to their first trimester blood pressure (BP) control. Group 1 includes women without a preceding history of $\mathrm{CH}$ presenting with BP of [?]140/90mmHg. Groups 2-4 have pre-pregnancy CH; in group 2 the $\mathrm{BP}$ is $<140 / 90 \mathrm{mmHg}$ without antihypertensive medication, in group 3 the $\mathrm{BP}$ is $<140 / 90 \mathrm{mmHg}$ with antihypertensive medication and in group 4 the $\mathrm{BP}$ is [?]140/90 $\mathrm{mmHg}$ despite antihypertensive medication. ${ }^{2}$ We have previously demonstrated that, across these four groups, the prevalence of superimposed PE is not uniformly distributed with group 4 having the highest rate of $27 \%$ compared to only $13 \%$ for group $1 .^{2}$ We hypothesised that this stratification reflects the severity of endothelial disease in women with $\mathrm{CH}$.

The pathophysiology of PE is thought to involve a tendency towards an anti-angiogenic state. ${ }^{3}$ Uteroplacental hypoxia in pregnancies destined to develop PE plays an important role in shifting the production in favour of the anti-angiogenic soluble fms like tyrosine kinase-1 (sFLT-1) at the expense of a reduction in the pro-angiogenic placental growth factor (PLGF). ${ }^{4}$ This has lead to considerable interest in their ability to predict PE, with PLGF already being incorporated into screening algorithms. ${ }^{5-7}$ However, it has been 
demonstrated both outside and in pregnancy that differences in the levels of angiogenic factors exist in patients with CH. ${ }^{8}, 9$ This could impact upon the predictive performance of PLGF and sFLT-1 and requires further clarification prior to their incorporation into screening models.

Outside of pregnancy, the pathophysiology of $\mathrm{CH}$ involves a coordinated interaction between the endothelium and circulating inflammatory mediators that include interleukin-6 (IL-6) and tumour necrosis factor alpha $(\mathrm{TNF}-\alpha) \cdot{ }^{10,11} \mathrm{TNF}-\alpha$ promotes both the endothelial expression of vascular cell adhesion molecule (VCAM $)^{10}$, an early event in the development of the atherosclerotic plaque, and the production of endothelin, a potent vasoconstrictor. ${ }^{12}$ TNF- $\alpha$, VCAM and endothelin all induce the production of IL- $6^{10,13}$, which through its effects on vascular remodelling, contributes to the chronic elevation of BP. ${ }^{11}$ Thus, these inflammatory mediators, as markers of endothelial dysfunction outside of pregnancy, have been identified as possible biomarkers, in addition to angiogenic factors, for the prediction of PE.

Our objective was to examine differences in angiogenic and inflammatory factors in women with $\mathrm{CH}$ at $11^{+0}-13^{+6}$ weeks according to BP control.

\section{METHODS}

This was a case-control study involving analysis of serum PLGF, sFLT-1, IL-6, TNF- $\alpha$, endothelin and VCAM levels in stored samples $\left(-80^{\circ} \mathrm{C}\right)$ obtained at 11-13 weeks' gestation from 650 singleton pregnancies complicated by $\mathrm{CH}$ and 142 normotensive control subjects who attended King's College Hospital (London, UK) for pregnancy care between January 2011 and September 2018. Gestational age was determined from measurement of fetal crown-rump length. ${ }^{14}$

\section{Patient involvement}

Participants were invited to provide feedback on their pregnancy care at King's College Hospital and to be contacted with the study findings. Otherwise, they were not involved in obtaining funding, study design or in the data analyses.

\section{Inclusion and exclusion criteria}

The inclusion criteria for this study were singleton pregnancies resulting in the live birth or stillbirth of phenotypically normal babies at $>24$ weeks' gestation. Pregnancies with fetal aneuploidies or major defects diagnosed in the antenatal or neonatal period and pregnancies ending in miscarriage at $<24$ week's gestation were excluded.

For every 5 cases with $\mathrm{CH}$, we selected approximately 1 control from uncomplicated pregnancies that resulted in the live birth of phenotypically normal neonates that were matched to the cases for storage time of maternal serum and racial origin, because the incidence of chronic hypertension is 3 times higher in Black than white women.

\section{Main outcome measures}

Women were classified as CH if they had pre-pregnancy hypertension or had BP [?]140/90 mmHg on two consecutive clinical visits prior to 20 weeks' gestation in the absence of renal or liver disease. ${ }^{15}$ Preeclampsia was defined according to the International Society for the Study of Hypertension in Pregnancy (ISSHP) ${ }^{15}$ as the presence of hypertension along with at least one of the following: renal involvement (proteinuria [?] $300 \mathrm{mg} / 24 \mathrm{~h}$ and/or creatinine [?] $90 \mu \mathrm{mol} / \mathrm{L}$ or $1 \mathrm{mg} / \mathrm{dL}$ ), liver impairment (transaminases $>70 \mathrm{IU} / \mathrm{L}$ ), neurological complications (e.g. eclampsia), thrombocytopenia (platelet count <150,000/ $\mu \mathrm{L}$ ).

\section{Assay analysis}

Angiogenic factors

Maternal serum concentrations of PLGF and sFLT-1 in pg/mL were measured from thawed samples using an automated biochemical analyser (BRAHMS KRYPTOR compact PLUS; Thermo Fisher Scientific, Hennigsdorf, Germany). In this analyser, the inter-assay coefficients of variation for the low and high concentrations 
were $22 \%$ and $5 \%$ for PlGF and $5 \%$ and $5 \%$ for sFLT-1, respectively. Assays cover a measurement range from 3.6-7000 pg/mL for PlGF and from 22-90,000pg/mL for sFLT-1.

\section{Inflammatory mediators}

$T N \Phi-a$ a $\delta I \Lambda-6$

Serum concentrations of TNF- $\alpha$ and IL-6 were determined using the Meso Scale Proinflammatory 2 Plex (Mesoscale Discovery, MD, USA). The detection limits were 0.04 and $0.06 \mathrm{ng} / \mathrm{L}$ for TNF- $\alpha$ and IL-6, respectively, with a measurement range from 0.10 to $1.75 \mathrm{ng} / \mathrm{L}$ and 0.16 to $27.2 \mathrm{ng} / \mathrm{L}$ for TNF- $\alpha$ and IL-6, respectively. The intra-assay coefficients of variation for the low and high concentrations were $10.1 \%$ and $6.1 \%$ for TNF- $\alpha$ and $4.5 \%$ and $3.6 \%$ for IL- 6 . The inter-assay coefficients of variation for the low and high concentrations were $6.2 \%$ and $7.2 \%$ for TNF- $\alpha$ and $7.3 \%$ and $5.2 \%$ for IL-6.

\section{Endothelin}

Serum concentrations of endothelin were determined using the Quantikine Endothelin-1 ELISA kit (Biotechne, R\&D Systems, Oxon, UK). The detection limit was $0.09 \mathrm{ng} / \mathrm{L}$ with a measurement range of 0.45 to $2.00 \mathrm{ng} / \mathrm{L}$. The intra-assay coefficients of variation for the low and high concentrations were $4.0 \%$ and $1.9 \%$. The inter-assay coefficients of variation for the low and high concentrations were $7.6 \%$ and $5.3 \%$.

\section{$V C A M$}

Serum concentrations of VCAM were determined using the Quantikine VCAM ELISA kit (Biotechne, R\&D Systems, Oxon, UK). The detection limit was $0.60 \mu \mathrm{g} / \mathrm{L}$ with a measurement range 349 to $991 \mu \mathrm{g} / \mathrm{L}$. The intra-assay coefficients of variation for the low and high concentrations were $2.3 \%$ and $3.6 \%$. The inter-assay coefficients of variation for the low and high concentrations were $7.8 \%$ and $5.5 \%$.

\section{Statistical analysis}

Normality of the distribution of continuous variables was assessed by the Kolmogorov-Smirnov test. Numerical data were expressed as mean and standard deviation (SD) or median and interquartile range (IQR) for normally and non-normally distributed data, respectively.

The distribution of sFLT-1, PLGF, sFLT-1/PLGF ratio, IL-6, TNF- $\alpha$, endothelin and VCAM were logarithmically transformed to approximate Gaussian distribution. All with the exception of log TNF- $\alpha$ were converted into multiples of the expected median (MoM) after adjustment for maternal characteristics. Log TNF- $\alpha$ was not converted to MoMs as there were no significant independent maternal contributions identified in the multiple regression model. Between group comparisons were made by the ANOVA or the KruskalWallis test, with Bonferroni correction for post-hoc analysis, for normally and non-normally distributed data, respectively. The Chi-square test was used to compare categorical variables.

Statistical analysis was performed using SPSS (Version 24; SPSS Inc, Chicago,IL).

\section{RESULTS}

\section{Population characteristics}

The inclusion criteria for women with $\mathrm{CH}$ were met by 650 pregnancies and were classified as group $1(\mathrm{n}=81)$, group $2(\mathrm{n}=199)$, group $3(\mathrm{n}=221)$ and group $4(\mathrm{n}=149)$. The control group consisted of 142 normotensive women. Maternal and pregnancy characteristics of the four groups of women with $\mathrm{CH}$ and the control group are compared in Table 1. In the total cohort of women with $\mathrm{CH}$, compared with controls, maternal age, weight and systolic and diastolic blood pressure at $11-13^{+6}$ weeks gestation and the incidence of previous and/or family history of preeclampsia were higher. Approximately $93 \%$ of women in groups 3 and 4 were taking one anti-hypertensive medication with $7 \%$ taking 2 or more. In groups 3 and 4, compared to group 2, there was a significantly higher weight, systolic blood pressure, incidence of Black racial origin and previous PE. In groups 2 and 3, compared to group 1, systolic and diastolic pressure were lower. Women of group 2 were more likely to be younger and nulliparous when compared to group 3 and with a lower diastolic blood 
pressure when compared to group 4 . Those of group 1 were also more likely to weigh more and be nulliparous when compared to groups 2 and 3, respectively, and with a lower systolic blood pressure compared to group 4. Groups 1 and 2 had a significantly lower rate of preterm PE and higher gestation at delivery when compared to groups 3 and 4 and a significantly lower incidence of SGA neonate with higher birth weight centile when compared to group 3 , alone.

\section{Serum PLGF, sFLT-1 and sFLT-1 / PLGF ratio}

Creation of multiples of the median using data from controls

In the multiple regression model for log sFLT-1, significant independent contributions were provided by age, weight, Black racial origin, parity and history of previous preeclampsia $\left(\mathrm{p}=<0.001, \mathrm{R}^{2}=0.251\right)$ : Log sFLT-1 expected $=3.142305+0.007276 \mathrm{x}$ age (in years) $-0.003325 \mathrm{x}$ weight $($ in $\mathrm{kg})+0.102288 \mathrm{x}$ Black racial origin $-0.172146 \mathrm{x}$ multiparity $+0.158197 \mathrm{x}$ previous preeclampsia.

In the multiple regression model for log PLGF, significant independent contributions were provided by age, weight and Black racial origin $\left(p=<0.001, R^{2}=0.207\right)$ : Log PLGF expected $=1.524666+0.008217 \times$ age (in years) $-0.002721 \mathrm{x}$ weight (in $\mathrm{kg}$ ) $+0.170813 \times$ Black racial origin.

In the multiple regression model for log sFLT-1/PLGF ratio, significant independent contributions were provided by parity alone $\left(\mathrm{p}=0.001, \mathrm{R}^{2}=0.082\right)$ : Log $\mathrm{sFLT}-1 / \mathrm{PLGF}$ ratio expected $=1.486950-0.151459$ if parous.

Comparison of multiples of the median between groups

Groups 2-4, compared to the control group, had significantly lower mean levels of PLGF and groups 2 and 3 had significantly lower mean levels of sFLT-1 (Table 2, Figure 1). Women with CH, compared to the control group had a higher sFLT-1/PIGF ratio, but the differences did not reach statistical significance (Table 2, Figure 1). Between the four groups of women with $\mathrm{CH}$, there were no significant differences in the levels of sFLT-1, PlGF and sFLT-1/PlGF ratio (Table 2, Figure 1).

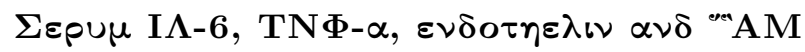

Creation of multiples of the median using data from controls

In the multiple regression model for log VCAM, significant independent contributions were provided by Black racial origin $\left(\mathrm{p}=<0.01, \mathrm{R}^{2}=0.067\right)$ : Log VCAM expected $=2.754330-0.051222 \times$ Black racial origin.

In the multiple regression model for log IL-6, significant independent contributions were provided by weight and 'Other' racial origin $\left(\mathrm{p}=<0.001, \mathrm{R}^{2}=0.125\right)$ : Log IL-6 expected $=5.496581+0.004763 \mathrm{x}$ weight (in $\mathrm{kg})+0.309845 \mathrm{x}$ 'Other' racial origin.

In the multiple regression model for log endothelin, significant independent contributions were provided by Black racial origin and parity $\left(\mathrm{p}=<0.001, \mathrm{R}^{2}=0.138\right)$ : Log endothelin expected $=2.297770+0.076133 \mathrm{x}$ Black racial origin $+0.073548 \times$ parity.

In the multiple regression model for $\log \mathrm{TNF}-\alpha$, there were no significant independent contributions provided by maternal characteristics.

Comparison of multiples of the median between groups

Groups 1 and 4, compared to the control group, had significantly higher mean levels of endothelin and, only group 4 had significantly lower mean levels of IL-6 (Table 2, Figure 2). There were no significant differences between the control group and women with $\mathrm{CH}$ in TNF- $\alpha$ or VCAM (Table 2, Figure 2). Between the four groups of women with $\mathrm{CH}$, there were no significant differences in the levels of IL-6, TNF- $\alpha$, endothelin or VCAM (Table 2, Figure 2).

\section{DISCUSSION}

\section{Main findings}


The findings of this study demonstrate that at $11-13^{+6}$ weeks' gestation in the $\mathrm{CH}$ groups, compared to controls, serum PLGF was reduced in those with pre-pregnancy CH (groups 2-4) and serum sFLT-1 was reduced in those with normal BP (groups 2-3). In addition, serum endothelin was increased in women with $\mathrm{CH}$ who are hypertensive at presentation (groups 1 and 4) but IL-6 was reduced only in those that remained hypertensive despite antihypertensive medications (group 4).

\section{Strengths and limitations}

The strengths of this study are examination of a large population of women with $\mathrm{CH}$ recruited in the first trimester of pregnancy. Exclusion of women with underlying renal and/or liver disease has reduced the potential for bias in the diagnosis of PE. A limitation of this study is the absence of longitudinal measurements of angiogenic and inflammatory factors throughout and outside of pregnancy to correlate with possible changes that can occur during pregnancy and the postnatal period.

\section{Interpretation of findings}

We hypothesised that the four subgroups of women with $\mathrm{CH}$ represent different stages of the disease. Studies in non-pregnant individuals have demonstrated that in the progression from normal blood pressure to mild and then severe hypertension there is increasing endothelial dysfunction. Groups 1 and 2 are likely to represent the early phase of $\mathrm{CH}$ with mild endothelial dysfunction but sufficient ability to achieve physiological adaptation to pregnancy with normalisation of BP in the latter group. Groups 3 and 4 may represent moderate to severe endothelial dysfunction with less capacity to achieve early vasodilation and normalisation in BP without antihypertensive medications. We further postulated that the severity of underlying endothelial disease, as represented by the four groups, might be reflected in the first trimester angiogenic and inflammatory profiles.

\section{Angiogenic factors}

During pregnancy, it is well established that, in the general obstetric population, PLGF is reduced from as early as the first trimester in pregnancies later complicated by PE and sFLT-1 is increased up to five weeks prior to the clinical onset of the disease. ${ }^{3}$ However, little is known as to the impact of $\mathrm{CH}$ on first trimester serum PLGF and sFLT-1.

In women with $\mathrm{CH}$, it could be anticipated that the degree of early placental hypoxia would be greater than in normotensive pregnancies due to pre-existing endothelial disease with a consequent higher production of sFLT-1 and lower PLGF. The latter is in keeping with our finding, along with other studies, of significantly lower first trimester serum PLGF in women with $\mathrm{CH} .{ }^{16}$ However, we were unable to demonstrate any differences in first trimester serum PLGF between the four groups of women with $\mathrm{CH}$ despite the differences in the rates of, particularly preterm, PE. It has previously been shown that, in women with $\mathrm{CH}$, the distribution of birth weight adjusted for gestational age at delivery is skewed to the left of the distribution for uncomplicated pregnancies and that there is an approximate 2 -fold increase in the risk of having a small for gestational age infant even in the absence of PE. ${ }^{1}$ Along with our findings, this suggests that $\mathrm{CH}$ is associated with impaired placentation irrespective of the development of $\mathrm{PE}$.

Our finding of lower sFLT-1 in women with CH who had well-controlled BP only was difficult to interpret. We postulated that an impaired placenta, as expected in women with $\mathrm{CH}$, would have less capacity to produce sFLT-1. However, should this be the case, one would expect low sFLT-1 in all four groups. It is likely that, although the risk of $\mathrm{PE}$ is not homogenous across all women with $\mathrm{CH}$, alteration in first trimester serum sFLT-1 does not have a significant role in the later development of PE and / or, alternatively, our study was underpowered to detect subtle differences between the four groups. Although there are studies examining first trimester serum sFLT-1 in women with $\mathrm{CH}$, none of them included normotensive controls for comparison..$^{9,17}$

\section{Inflammatory mediators}

Outside of pregnancy, increased serum IL-6 and TNF- $\alpha$ has been reported in patients with CH. In one study, 
the prevalence of hypertension was double in those in the upper quartile of IL- 6 and TNF- $\alpha$ when compared to those in the lower quartile. ${ }^{18}$ Two smaller studies further support a role for TNF- $\alpha$ in the pathophysiology of hypertension by reporting elevated TNF- $\alpha$ in those with $\mathrm{CH}$ compared to those without. ${ }^{19,}{ }^{20} \mathrm{TNF}-\alpha$ has been shown to induce structural as well as functional alterations in endothelial cells, enhances the production of endothelin and other inflammatory mediators, including IL-6 and VCAM, that ultimately lead to atherosclerotic plaque formation and progression. ${ }^{12,}{ }^{21}$ In addition, the increases in serum IL- 6 , TNF- $\alpha$, endothelin and VCAM have been correlated to the degree of cardiovascular risk in patients with $\mathrm{CH}$ and, thus, have emerged as predictors of cardiovascular morbidity. ${ }^{22}$

During pregnancy, there is extensive literature to support a 2-3 fold increase in the production of IL-6, TNF- $\alpha$, endothelin and VCAM in pregnancies already complicated with PE. ${ }^{23,}{ }^{24}$ It has been proposed that, similar to outside of pregnancy, these inflammatory mediators lead to the development of atheroscleroticlike lesions. ${ }^{25}$ Within the feto-placental circulation, these lesions result in placental hypoxia and, within the systemic circulation, result in endothelial dysfunction and the maternal syndrome of PE. ${ }^{26}$ However, it remains to be clarified as to whether this inflammatory process predates the placental impairment or whether it is a consequence of it.

Our work has demonstrated that, in women with $\mathrm{CH}$, first trimester alterations in IL-6 and endothelin exist. We have shown that women with $\mathrm{CH}$ who are hypertensive have elevated first trimester serum endothelin when compared to normotensive controls. Women in group 4 who are taking antihypertensive medications are likely to have had a longer duration of disease than those in group 1 who are previously undiagnosed. Along with our findings, this supports the existing literature where a positive correlation between levels of endothelin and mean arterial pressure but not duration of disease has been demonstrated. ${ }^{23,} 27$

Endothelin is also known to increase the expression of other inflammatory cytokines such as IL-6, another biomarker of endothelial disease. ${ }^{13}$ The finding of lower first trimester serum IL- 6 for group 4 only when compared to normotensive controls was unexpected. In normal pregnancy, current literature has demonstrated a doubling in the maternal serum concentration of IL-6 when compared to non-pregnant controls due to trophoblastic production in the early first trimester. ${ }^{28}$ It has been suggested that IL- 6 may have a major role in the normal paracrine regulation of placental development and hormone production. ${ }^{28}$ We propose that in women with $\mathrm{CH}$, particularly those with more severe disease as in group 4, there is less capacity of the impaired placenta to up-regulate its production of IL-6, as with sFLT-1. In contrast, first trimester maternal serum concentration of endothelin is approximately halved when compared to non-pregnant controls and is considered to be a protective mechanism against its vasoconstrictive properties. ${ }^{29}$ Thus, the endothelin levels measured are a reflection of maternal systemic production rather than a trophoblastic contribution, as with IL-6.

\section{Conclusion}

In women with $\mathrm{CH}$, when compared to normotensive controls, there are differences in the first trimester angiogenic and inflammatory profiles according to BP control and use of antihypertensive medications. Further evaluation is needed as to whether these differences are clinically useful in the stratification of care.

\section{Disclosures of interest}

The authors report no conflict of interest

\section{Contribution of authorship}

DN was involved in the collection and organisation of data, statistical analysis and writing the first draft of the manuscript. KHN and NK were involved in the conception of the study and editing the manuscript. In addition, NK was involved in the collection and organisation of data and statistical analysis.

\section{Ethics approval}

Written informed consent was obtained from women who agreed to participate in the study on early prediction of pregnancy complications, approved by the National Health Service Health Research Authority, Dulwich 
Research Ethics Committee (REC reference 02-03-033) on $17^{\text {th }}$ February 2017.

\section{Sources of funding}

The study was funded by the Fetal Medicine Foundation (Registered charity No. 1037116). The reagents and equipment for the measurement of serum placental growth factor and soluble fms-like tyrosine kinase-1 were provided by Thermo Fisher Scientific. These bodies had no involvement in the study design; in the collection, analysis and interpretation of data; in the writing of the report; and in the decision to submit the article for publication.

\section{REFERENCES}

1. Panaitescu AM, Syngelaki A, Prodan N, Akolekar R, Nicolaides KH. Chronic hypertension and adverse pregnancy outcome: a cohort study. Ultrasound Obstet Gynecol . 2017;50(2):228-35.

2. Nzelu D, Dumitrascu-Biris D, Nicolaides KH, Kametas NA. Chronic hypertension: first-trimester blood pressure control and likelihood of severe hypertension, preeclampsia, and small for gestational age.Am $J$ Obstet Gynecol . 2018;218(3):337.e1-.e7.

3. Chaiworapongsa T, Chaemsaithong P, Yeo L, Romero R. Pre-eclampsia part 1: current understanding of its pathophysiology. Nat Rev Nephrol . 2014;10(8):466-80.

4. Brosens I, Pijnenborg R, Vercruysse L, Romero R. The "Great Obstetrical Syndromes" are associated with disorders of deep placentation. Am J Obstet Gynecol . 2011;204(3):193-201.

5. Akolekar R, Zaragoza E, Poon L, Pepes S, Nicolaides K. Maternal serum placental growth factor at 11+ 0 to $13+6$ weeks of gestation in the prediction of pre-eclampsia. Ultrasound in Obstetrics and Gynecology . 2008;32(6):732-9.

6. O'Gorman N, Wright D, Poon LC, Rolnik DL, Syngelaki A, Wright A, et al. Accuracy of competing-risks model in screening for pre-eclampsia by maternal factors and biomarkers at 11-13 weeks' gestation. Ultrasound Obstet Gynecol . 2017;49(6):751-5.

7. Wright A, Wright D, Syngelaki A, Georgantis A, Nicolaides KH. Two-stage screening for preterm preeclampsia at 11-13 weeks' gestation.Am J Obstet Gynecol . 2019;220(2):197.e1-.e11.

8. Felmeden DC, Spencer CG, Blann AD, Beevers DG, Lip GY. Physical activity in relation to indices of endothelial function and angiogenesis factors in hypertension: a substudy of the Anglo-Scandinavian Cardiac Outcomes Trial (ASCOT). J Intern Med . 2003;253(1):81-91.

9. Perni U, Sison C, Sharma V, Helseth G, Hawfield A, Suthanthiran M, et al. Angiogenic factors in superimposed preeclampsia: a longitudinal study of women with chronic hypertension during pregnancy. Hypertension . 2012;59(3):740-6.

10. Dinarello CA. Inflammatory cytokines: interleukin-1 and tumor necrosis factor as effector molecules in autoimmune diseases. Curr Opin Immunol . 1991;3(6):941-8.

11. Ikeda U, Ikeda M, Oohara T, Oguchi A, Kamitani T, Tsuruya Y, et al. Interleukin 6 stimulates growth of vascular smooth muscle cells in a PDGF-dependent manner. Am J Physiol . 1991;260(5 Pt 2):H1713-7.

12. Schiffrin EL. Endothelin: potential role in hypertension and vascular hypertrophy. Hypertension . $1995 ; 25(6): 1135-43$.

13. Ishizuka T, Takamizawa-Matsumoto M, Suzuki K, Kurita A. Endothelin-1 enhances vascular cell adhesion molecule-1 expression in tumor necrosis factor alpha-stimulated vascular endothelial cells. Eur J Pharmacol . 1999;369(2):237-45.

14. Robinson H, Fleming J. A critical evaluation of sonar "crown-rump length" measurements. BJOG: An International Journal of Obstetrics $\&$ Gynaecology . 1975;82(9):702-10. 
15. Tranquilli A, Dekker G, Magee L, Roberts J, Sibai B, Steyn W, et al. The classification, diagnosis and management of the hypertensive disorders of pregnancy: a revised statement from the ISSHP.Pregnancy Hypertens . 2014;4(2):97-104.

16. Panaitescu AM, Akolekar R, Kametas N, Syngelaki A, Nicolaides KH. Impaired placentation in women with chronic hypertension who develop pre-eclampsia. Ultrasound Obstet Gynecol . 2017;50(4):496-500.

17. Powers RW, Jeyabalan A, Clifton RG, Van Dorsten P, Hauth JC, Klebanoff MA, et al. Soluble fms-Like tyrosine kinase 1 (sFlt1), endoglin and placental growth factor (PlGF) in preeclampsia among high risk pregnancies. PloS one . 2010;5(10):e13263.

18. Bautista LE, Vera LM, Arenas IA, Gamarra G. Independent association between inflammatory markers (C-reactive protein, interleukin-6, and TNF-alpha) and essential hypertension. J Hum Hypertens . 2005;19(2):149-54.

19. Furumoto T, Saito N, Dong J, Mikami T, Fujii S, Kitabatake A. Association of cardiovascular risk factors and endothelial dysfunction in japanese hypertensive patients: implications for early atherosclerosis. Hypertens Res . 2002;25(3):475-80.

20. Ito H, Ohshima A, Tsuzuki M, Ohto N, Takao K, Hijii C, et al. Association of serum tumour necrosis factor-alpha with serum low-density lipoprotein-cholesterol and blood pressure in apparently healthy Japanese women. Clin Exp Pharmacol Physiol . 2001;28(3):188-92.

21. Yoshizumi M, Perrella MA, Burnett JC, Jr., Lee ME. Tumor necrosis factor downregulates an endothelial nitric oxide synthase mRNA by shortening its half-life. Circ Res . 1993;73(1):205-9.

22. O'Brien KD, Allen MD, McDonald TO, Chait A, Harlan JM, Fishbein D, et al. Vascular cell adhesion molecule-1 is expressed in human coronary atherosclerotic plaques. Implications for the mode of progression of advanced coronary atherosclerosis. J Clin Invest . 1993;92(2):945-51.

23. Wolff K, Nisell H, Carlstrom K, Kublickiene K, Hemsen A, Lunell NO, et al. Endothelin-1 and big endothelin-1 levels in normal term pregnancy and in preeclampsia. Regul Pept . 1996;67(3):211-6.

24. Szarka A, Rigo J, Jr., Lazar L, Beko G, Molvarec A. Circulating cytokines, chemokines and adhesion molecules in normal pregnancy and preeclampsia determined by multiplex suspension array. BMC Immunol . 2010;11:59.

25. Labarrere CA, DiCarlo HL, Bammerlin E, Hardin JW, Kim YM, Chaemsaithong P, et al. Failure of physiologic transformation of spiral arteries, endothelial and trophoblast cell activation, and acute atherosis in the basal plate of the placenta. Am J Obstet Gynecol . 2017;216(3):287.e1-.e16.

26. Staff AC, Johnsen GM, Dechend R, Redman CWG. Preeclampsia and uteroplacental acute atherosis: immune and inflammatory factors. J Reprod Immunol . 2014;101-102:120-6.

27. Widimsky J, Jr., Horky K, Dvorakova J. Plasma endothelin-1,2 levels in mild and severe hypertension. J Hypertens Suppl . 1991;9(6):S194-5.

28. Opsjln SL, Wathen NC, Tingulstad S, Wiedswang G, Sundan A, Waage A, et al. Tumor necrosis factor, interleukin-1, and interleukin-6 in normal human pregnancy. Am J Obstet Gynecol . 1993;169(2 Pt $1): 397-404$.

29. Lygnos MC, Pappa KI, Papadaki HA, Relakis C, Koumantakis E, Anagnou NP, et al. Changes in maternal plasma levels of VEGF, bFGF, TGF-beta1, ET-1 and sKL during uncomplicated pregnancy, hypertensive pregnancy and gestational diabetes. In Vivo . 2006;20(1):157-63.

\section{Hosted file}

Table 1_ 1st TM angiogenic \& inflammatory markers.pdf available at https://authorea.com/ users/326694/articles/492294-first-trimester-angiogenic-and-inflammatory-factors-in- 
women-with-chronic-hypertension-and-impact-of-blood-pressure-control-a-case-controlstudy

\section{Hosted file}

Table 2_1st TM angiogenic \& inflammatory markers.pdf available at https://authorea.com/users/ 326694/articles/492294-first-trimester-angiogenic-and-inflammatory-factors-in-womenwith-chronic-hypertension-and-impact-of-blood-pressure-control-a-case-control-study
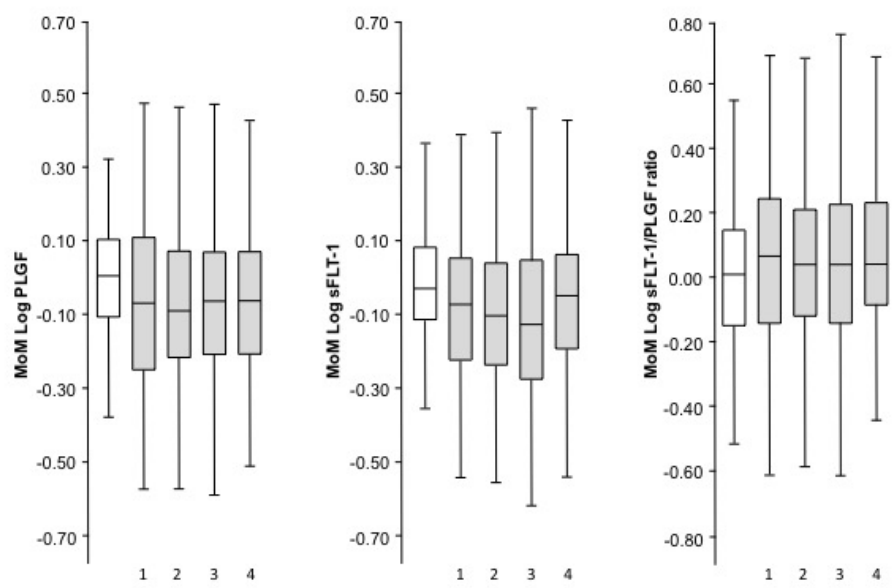

Figure 1: Serum levels of MoM Log PLGF, MoM Log sFLT-1 and MoM Log SFLT-1/PLGF ratio in controls (white) and groups 1-4 (grey) of women with CH.

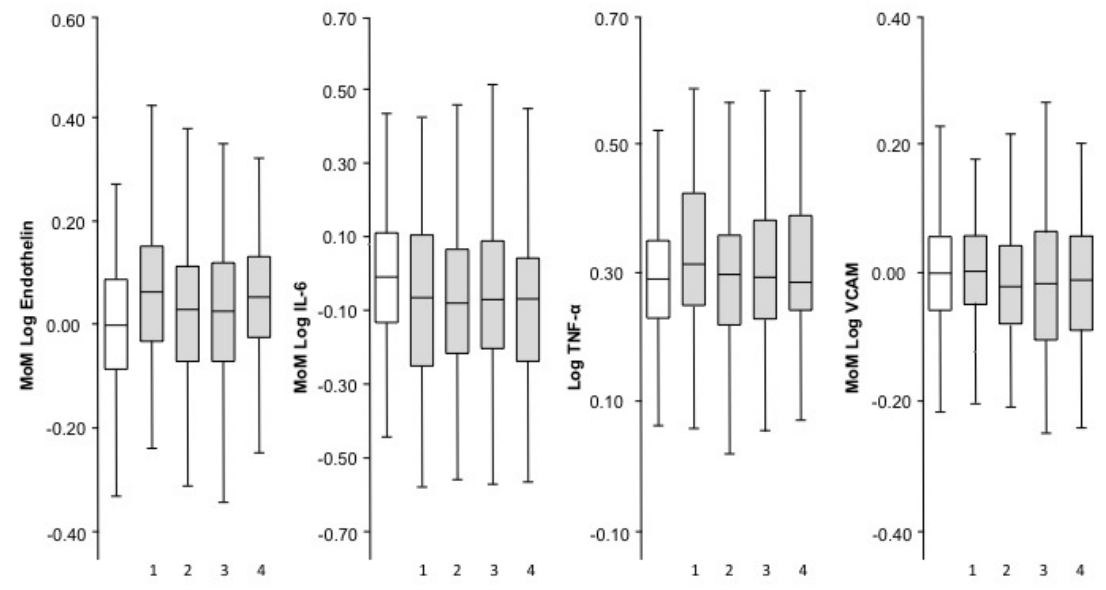

Figure 2: Serum levels of MoM Log endothelin, MoM Log IL-6, Log TNF- $\alpha$ and MoM Log VCAM in controls (white) and groups 1-4 (grey) of women with CH. 